\title{
IDENTIFICATION AND CHARACTERISATION OF A CDNA CLONE FOR CYTOSOLIC GLYCERALDEHYDE-3-PHOSPHATE DEHYDROGENASE IN BARLEY
}

by

\author{
JAN CHOJECKI
}

\begin{abstract}
Department of Physiology, Carlsberg Laboratory, Gamle Carlsberg Vej 10, DK-2500 Copenhagen Valby
\end{abstract}

Keywords: DNA sequence, germination

\begin{abstract}
The nucleotide sequence of a cDNA clone for barley glyceraldehyde-3-phosphate dehydrogenase (GAPDH) has been determined. The cDNA is 1147 base pairs long, encoding 305 amino-acids of the GAPDH polypeptide. Comparison of the amino acid sequence, predicted from the DNA sequence, with GAPDH sequences available for other organisms reveals a high degree of sequence conservation, particularly in regions of supposed importance for tertiary structure and activity. Hybridisation of the cDNA insert to barley genomic DNA indicates that there is a single copy of the gene per haploid genome. The expression of the GAPDH gene in the scutellum during germination, as detected by RNA hydridisation analysis, probably reflects the involvement of the enzyme at key steps in both the glycolytic and gluconeogenic pathways.
\end{abstract}

\section{INTRODUCTION}

The initial aim of this investigation was to obtain and identify cDNA clones to use as probes to study gene expression during barley development, in general, and during seed germination, in particular. This paper describes the identification and preliminary analysis of a cDNA clone for cytosolic, NAD-specific glyceraldehyde-3-phosphate dehydrogenase (EC 1.2.1.12).

The enzyme plays a central role in carbohydrate metabolism, catalysing an important step in both the glycolytic and gluconeogenic pathways.

\section{MATERIALS PND METHODS}

2.1. CDNA library and DNA sequence analysis

The cDNA library was constructed using the method of WiCKENS et al. (18) from mRNA isolated from scutella of Nudinka barley seeds two days after the onset of germination. The library was kindly provided by Drs. A. BRANDT and G. FINCHER. The library was partially characterised by hybridisation to reverse transcribed, size-fractionated mRNA yielding by in-vitro translation polypeptides of between 25 and 35 kiloDaltons. Clones thus selected were subjected to partial sequence analysis, by subcloning into bacteriophage M13mp8 and sequencing by the dideoxynucleotide chain termination method (15). Amino acid sequences predicted from open reading frames were compared with published sequence data using the Protein Identification Resource computer programme and database (NBRF,Georgetown University Medical Centre, 3900 Reservoir Road, N.W., Washington DC 20007).

Abbreviations: $b p=$ base pair; GAPDH $=$ glyceraldehyde-3-phosphate dehydrogenase; $k b p=$ kilobase pairs. 


\section{J. CHOJECKI: Barley GAPDH cDNA}

1 50

GTC AAC GAC CCG TTC ATC ACC ACC GAC TAC ATG ACC TAC ATG TTC AAG TAT GAC ACT GTC CAC GGA VAL ASN ASP PRO PHE ILE THR THR ASP TYR MET THR TYR MET PHE LYS TYR ASP THR VAL HIS GLY 30

100

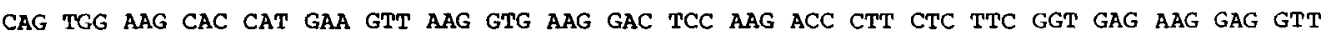
GLN TRP LYS HIS HIS GLU VAL LYS VAL LYS ASP SER LYS THR LEU LEU PHE GLY GLU LYS GLU VAL 150

GCT GTG TTT GGT TGC AGA AAC CCC GAG GAG ATT CCA TGG GCC GCT GCT GGT GCT GAG TAC GTT GTG ALA VAL PHE GLY CYS ARG ASN PRO GLU GLU ILE PRO TRP ALA ALA ALA GLY ALA GLU TYR VAL VAL

200

250

GAG TCC ACC GGT GTT TTC ACT GAC AAG GAC AAG GCT GCA GCT CAC ATT AAG GGT GGT GCC AAG AAG GLU SER THR GLY VAL PHE THR ASP LYS ASP IYS ALA ALA ALA HIS ILE LYS GLY GLY ALA LYS LYS

300

GTC ATC ATT TCT GCT CCC AGC AAG GAC GCT CCC ATG TTT GTC TGT GGT GTC AAC GAG AAG GAA TAC VAL ILE ILE SER ALA PRO SER LYS ASP ALA PRO MET PHE VAL CYS GLY VAL ASN GLU LYS GLU TYR

AAG TCA GAC ATC GAC ATT GTC TCC AAT GCT AGC TGC ACC ACC AAC TGT CCT GCT CCT CTT GCT AAG LYS SER ASP ILE ASP ILE VAL SER ASN ALA SER CYS THR THR ASN CYS PRO ALA PRO LEU ALA LYS

400

450

159

GTT ATC AAT GAC AGG TTT GGC ATT GTT GAG GGT TTG ATG ACC ACT GTC CAT GCC ATG ACT GCT ACC VAL ILE ASN ASP ARG PHE GLY ILE VAL GLU GLY LEU MET THR THR VAL HIS ALA MET THR ALA THR 500

181

CAG AAG ACT GTT GAT GGT CCT TCA AGC AAG GAC TGG AGA GGT GGA AGG GCT GCT AGC TTC AAC ATC GLN LYS THR VAL ASP GLY PRO SER SER LYS ASP TRP ARG GLY GLY ARG ALA ALA SER PHE ASN ILE

550

ATT CCA AGC AGC ACT GGT GCT GCA AAG GCC GTT GGC AAG GTG CTC CCA GAA CTT AAC GGA AAG TTG ILE PRO SER SER THR GLY ALA ALA LYS ALA VAL GLY LYS VAL LEU PRO GLU LEU ASN GLY LYS LEU

600

650

ACT GGA ATG GCC TTC CGT GTT CCC ACT GTT GAT GTT TCT GTT GTT GAT CTG ACT GTT AGA CTT GCC THR GLY MET ALA PHE ARG VAL PRO THR VAL ASP VAL SER VAL VAL ASP LEU THR VAL ARG LEU ALA

700

AAG CCA GCC ACC TAT GAG CAG ATT AAG GCT GCT ATC AAG GAG GAG TCT GAG GGA AAC CTC AAG GGC LYS PRO ALA THR TYR GLU GLN ILE LYS ALA ALA ILE LYS GLU GLU SER GLU GLY ASN LEU LYS GLY 750

ATT TTG GGT TAT GTC GAT GAG GAC CTT GTT TCC ACT GAC TTC CAG GGT GAC AGC AGG TCC AGC ATC ILE LEU GLY TYR VAL ASP GLU ASP LEU VAL SER THR ASP PHE GLN GLY ASP SER ARG SER SER ILE

850

TTT GAT GCC AAG GCC GGG ATT GCT CTG AAC GAC AAC TTT GTC AAG CTT GTC TCA TGG TAC GAC AAC PHE ASP ALA LYS ALA GLY ILE ALA LEU ASN ASP ASN PHE VAL LYS LEU VAL SER TRP TYR ASP ASN

900

GAG TGG GGa TAC AGC ACC CGT GTG GTC GaC CTC ATC CGC CAC ATG CAC AGC ACC Aag TAA ATGAGCC GLU TRP GLY TYR SER THR ARG VAL VAL ASP LEU ILE ARG HIS MET HIS SER THR LYS ******;

950

1000

AAAGCATGAAGATACAGGGAGTGTGGTTTGCCCCAGAGAAGAGAAGAGTGTACAACCTCTTCCGAGAATAAATTTTTGTATGGAATT 1050 1100

ATGGCAACTAAAAAAAACCTTTTATTGGATGATCCTGATGGTTGGTTGAGCTTAGCGGCTCACATTTTGGTGGTATTATGTACTTGC

TTGAACTAAATCATGAGTTATTTTCACTCATCGTCATGGGTTAAAAAAA

Figure 1. Nucleotide sequence of a cDNA clone encoding barley cytosolic GAPDH, and predicted amino acid sequence.

The amino acid sequence is numbered according to the system of HARRIS and WATERS (6); starred residues indicate probable insertions (see text). The putative polyadenylation signals are underlined. The stop codon is outlined. 


\subsection{DNA and RNA hybridisation}

Barley genomic DNA (7) was digested, separated on agarose gels and transferred to nitrocellulose paper (16). The DNA was then probed with cDNA inserts labelled with $\left(\alpha^{32} \mathrm{P}\right) \mathrm{dATP}$ by nicktranslation (14). RNA blots were probed with the appropriate single-stranded M13 subclone (8).

\section{RESULTS}

The barley GAPDH cDNA clone was identified on the basis of strong homology between the predicted amino acid sequence and sequences of GAPDH from pig, chicken, lobster (each 69\% homology), human (68\%), and yeast (67\%) - all contained in the PIR database. The cDNA is $1147 \mathrm{bp}$ in length and encodes 305 amino acids of the GAPDH polypeptide, with a 3' non-coding region of 230 nucleotides. The nucleotide sequence, the predicted amino acid sequence and the sequencing strategy are shown in Figures 1 and 2.

Probing mRNA from scutella from 5 day-germinated barley seeds in a Northern hybridisation analysis (Figure 3 ) showed that the cDNA represents an mRNA of approximately $1.6 \mathrm{~kb}$. The cloned cDNA therefore lacks about 450 nucleotides from the 5 ' end of the mRNA. A comparison with the amino acid sequences of GAPDH from other organisms reveals that the barley cDNA clone encodes the amino acids from position 30 to the carboxyterminal of the

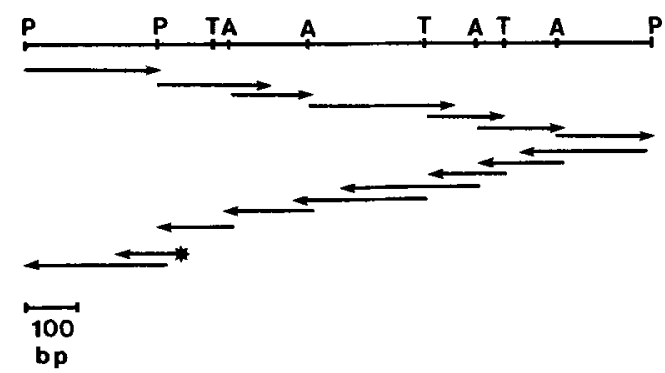

Figure 2. Strategy used for sequencing barley GAPDH cDNA.

The sequence originating from the $\star$ symbol was determined by priming on the double-stranded cDNA, using a specific 14'mer oligonucleotide synthesised by an Applied Biosystems 380A DNA synthesiser. P = Pst I, A = Alu I, T = Taq I restriction sites.

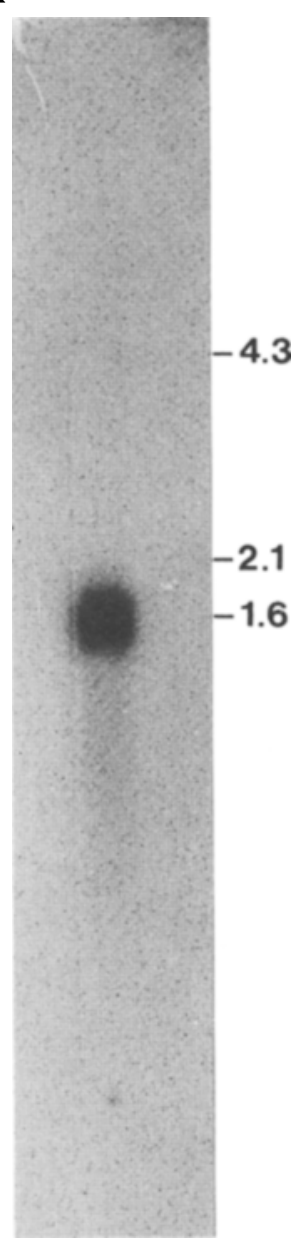

Figure 3. Northern hybridisation analysis.

Poly(A)+ RNA, prepared from scutella of barley seeds germinated for 5 days, was separated by electrophoresis in an agarose gel containing formamide, transferred to nitrocellulose paper and probed with the ( $\alpha^{32}$ P)dATP-labelled M13 subclone containing DNA complementary to GAPDH mRNA. Ribosomal 26S and 18S RNAs served as internal size markers (4.3 and $2.1 \mathrm{~kb}$ ), indicated to the right of the figure.

mature polypeptide (numbering system of HARRIS and WATERS (5)). Thus, the missing 5' nucleotides are expected to encode the $29 \mathrm{~N}$-terminal amino acids (87 nucleotides), and the 5' non-translated region of the mRNA.

Hybridisation of the nick-translated cDNA insert to barley genomic DNA from cv. Carlsberg II, digested with different restriction endo- 


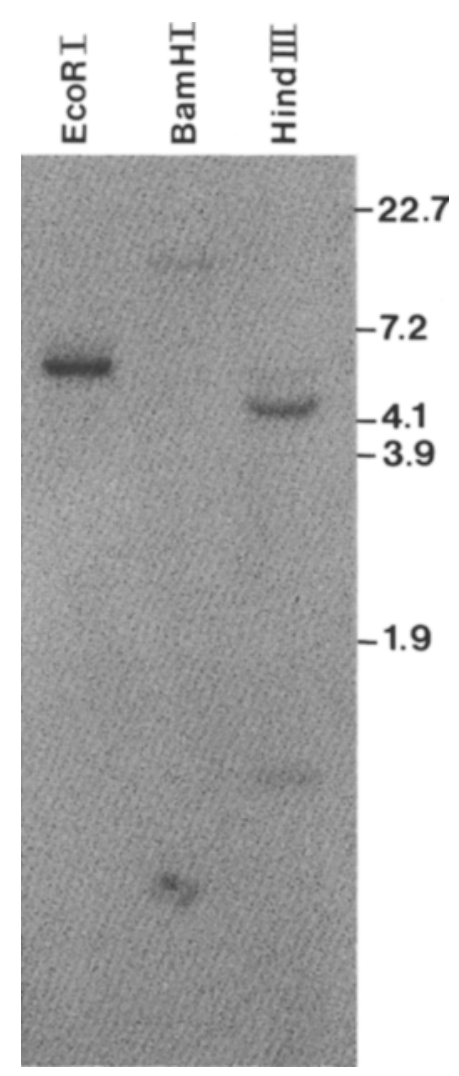

Figure 4. Southern hybridisation analysis.

Barley genomic DNA (cv. Carlsberg II), digested with different restriction endonucleases, and probed with the nick-translated GAPDH cDNA insert. Fragment sizes were estimated using the Hind III restriction fragments of $\lambda$-Wes $/ \lambda$-b as markers, indicated in $k b p$.

nucleases (Figure 4) indicates a single hybridising fragment in the Eco R 1 and Bam $\mathrm{H} 1$ digests, and two fragments in the Hind III digest. There is an internal Hind III site at nucleotides 834840 , generating the two Hind III fragments. These data suggest that there is only one copy of the GAPDH gene per haploid barley genome.

\section{DISCUSSION}

MARTIN and CERFF (10) have recently obtained and sequenced cDNA clones for cytosolic, NAD-specific GAPDH from mustard (Sinapis alba). The barley and mustard sequences are, within the coding region, $79 \%$ homologous at the nucleotide level and $86 \%$ homologous at the amino acid level. Both barley and mustard GAPDH cDNA sequences contain two triplet insertions which result in two extra residues in the predicted amino acid sequences compared with other known GAPDH proteins; lysine after residue 53 and glutamate after residue 68 (Figure 5). The high degree of conservation of amino acid sequences among GAPDHs from different organisms (10) presumably reflects a tight association between structure and function. The active GAPDH holoenzyme is made up by the association of four identical polypeptides, each with an approximate molecular weight of $36 \mathrm{kD}$ (for review, see ref. 6). The S-loops of each subunit are associated at the core of the holoenzyme $(1,17)$. The loop is formed by residues 178-201 (see Figure 5) and is one of the most highly conserved regions; only two residues ( 178 and 190) differ between barley and mustard and seven differ between barley and pig (4). The GAPDH amino acid sequence contains two functional domains; residues $0-148$ form the NAD-binding domain, while the remaining 186 amino acids form the catalytic domain, conferring substrate specificity and catalytic activity (11). The barley and mustard sequences have the same degree $(86 \%)$ of homology in both domains. However, there is a greater average sequence conservation in the catalytic domain (74\%) than in the NAD-binding domain $(61 \%)$ when the plant GAPDHs are compared with those of animals and yeast.

The residues surrounding the active site cysteine (residue 149) are also highly conserved; residues $145-156$ are identical in all NAD-spe-

Figure 5. Comparison of amino acid sequences of cytosolic GAPDH from barley, mustard (11), and pig (5).

The barley sequence is shown in full and the mustard and pig sequences are shown only where they differ from barley. The start of the sequence encoded by the barley cDNA is arrowed, up to which point only the mustard and pig sequences are compared. The widely conserved sequences of the active site (I) and the S-loop (II) are outlined. Stars in the pig sequence indicate the position of extra residues in the plant GAPDHs. Numbering of residues is as Figure 1. 


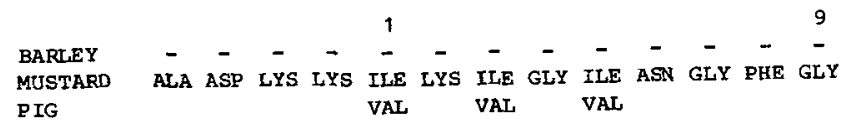

$\mathrm{B} \quad-\quad-\quad-$ - $-\quad-\quad-\quad-\quad-\quad-\quad-\quad-\quad-\quad-\quad-\quad-\quad--<-<-$

M PHE GLY ARG ILE GLY ARG LEU VAL ALA ARG VAL ILE LEU GIN ARG ASN ASP VAL GLU LEU VAL ALA

P THR ALA ALA PHE ASN SER GLY LYS ASP ILE

Y VAL ASN ASP PRO PHE ILE THR THR ASP TYR MET THR TYR MET PHE LYS TYR ASP THR VAL HIS GLY

$\begin{array}{lllll}\text { GLU } & & \text { SER } \\ \text { ILE ASP LEU HIS } & \text { VAL }\end{array}$

HTS GLU VAL UYS VAT LYS ASP SER IYS THR TEU LEU PHE GIY GLU TYS GLU VAL

ASN LEU Iys PHE * GLY THR ALA GLU GLY LEU VAl ILE aSP * ALA ILE

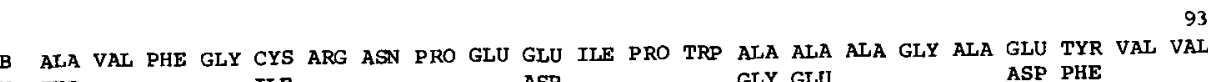

\begin{tabular}{lllllll}
$M$ & THR & ILE & ASP & GLY GLU & THR ALA \\
\hline & THR ILE GIN GLU ASP ALA ASN & LYS & GLY ASP
\end{tabular}

R 1115

B GLU SER THR GLY VAL PHE THR ASP LYS ASP LYS ALA ALA ALA HIS ILE LYS GLY GLY ALA LYS LYS

$\begin{array}{lllll}M & \text { LEU } & \text { LLY } & \text { LEU } & \text { ARG } \\ P & \text { THR MET GLU } & \text { GLY }\end{array}$

137

VAL ILE ILE SER ALA PRO SER LYS ASP ALA PRO MET PHE VAL CYS GLY VAL ASN GLU IYS GLU TYR

P ALA HET HIS GLU LYS

$\begin{array}{llll}M & \text { VAL } & \text { MET } & \text { HIS GLU LYS }\end{array}$

B LYS SER ASP ILE ASP ILE VAL SER ASN ALA SER CYS THR THR ASN CYS PRO ALA PRO ILU ALA LYS

LEU ASN
$P$ ASP ASN SER LEU LYS $\quad \begin{aligned} & \text { LEU } \\ & \text { LEU }\end{aligned}$

181

B VAL ILE ASN ASP ARG PHE GLY ILE VAL GLU GLY IEU MET THR THR VAL HIS ALA WET THAR ALA MHR

$M \quad$ HIS HIS SER ILE

II 203 \begin{tabular}{llllll}
$M$ & MET & & & & \\
$P$ & GLY & LEU & ASP & GLY & ALA GLN \\
\hline
\end{tabular}

TIER 225

ALA GLN

IIE ASP

247

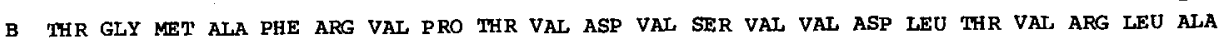

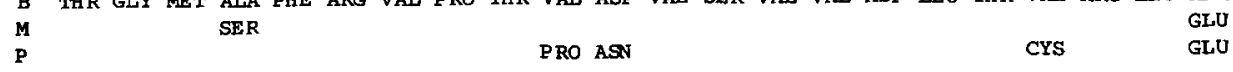

$\begin{array}{lllllllll}\text { B } & \text { LYS PRO ALA THR TYR GLU GLN } & \text { ILE LYS ALA ALA ILE LYS GLU GLU SER GLU GLY ASN LEU LYS GLY } \\ \text { M } & \text { ALA } & \text { ASP GLU } & \text { LYS } & & \text { GLN } & \text { LYS } \\ \text { P } & & \text { LYS } & \text { ASP ASP } & \text { LYS VAL VAL } & \text { GLN ALA } & & \text { PRO }\end{array}$

291

B ILE LEU GLY TYR VAL ASP GLU ASP LEU VAL SER THR ASP PHE GLN GLY ASP SER ARG SER SER ILE $\begin{array}{llllll}M & \text { THR GLU ASP VAL } & \text { VAL N } & \text { ASN } \\ P & \text { THR GLU ASP GLN VAL } & \text { CYS } & \text { ASN ASP SER THR HIS } & \text { THR }\end{array}$

313 B PHE ASP ALA Lys aLA GLY ILE ALA LEU ASN ASP ASN PHE VAL LYS LEU VAL SER TRP. TYR ASP ASN $\begin{array}{lllll}M & \text { SER } & & \\ P & \text { GLY } & \text { HIS }\end{array}$

B GLU TRP GLY TYR SER THR ARG VAL VAL ASP LEU ILE ARG GIS MET HIS SER THR LYS
SER LYS ALA

$\begin{array}{lllll}M & & \text { ILE } & \text { SER LYS ALA } \\ P & \text { PHE } & \text { ASN } & \text { MET VAL } & \text { ALA IYS GLU }\end{array}$ 
cific GAPDH sequences known, except for barley, where there is proline instead of leucine in position 154.

The codon usage (Figure 6) is not as biased as that of yeast GAPDH (6). Nevertheless, as in yeast there is a greater frequency of pyrimidine (69\%) than purine nucleotides in the third position of each codon, and a very low frequency (7\%) of codons ending in A. All 27 lysine residues (notably $9 \%$ of the 305 amino acid residues encoded by this cDNA) are coded by AAG triplets. The significance of biased codon usages is unclear, although it has been suggested (9) that control of translation rate of such mRNAs may be exerted through the relative levels of tRNA species.

The 3' non-coding region of the barley GAPDH cDNA clone shows no homology to the 3 ' region of its mustard counterpart (10), with the exception of a block of eight nuleotides (-TGTGGTTT-) located $29 \mathrm{bp}$, in barley, and 39 bp, in mustard, 3' from the termination codon (see Figure 1). Also indicated in Figure 1, is a sequence, AACTAAA, similar to the putative polyadenylation signal of eucaryotic mRNAs (13), lying $32 \mathrm{bp}$ upstream of a short region of poly(A) at the 3 ' limit of the cDNA insert. However, there is a AATAAA hexamer in the 3' non-coding region (bases 992-997), and it lies 24 nucleotides upstream of a stretch of 8 "A"s. It remains unclear whether the cDNA 3 ' of this region faithfully represents the GAPDH mRNA.

Southern hybridisation analysis (Figure 4) suggests that there is a single copy of the GAPDH gene per haploid barley genome. This contrasts with the very high copy number reported for rat and mouse (12), although only one of those copies is functional.

The cDNA for barley cytosolic GAPDH will enable a study to be made of the regulation of expression of the GAPDH gene during development. CERFF and KLOPPSTECH (2) demonstrated in a range of angiosperm species that mRNA levels for cytosolic GAPDH were unaffected by light - as might be expected for a ubiquitous "house-keeping" enzyme. In barley, the mRNA for cytosolic GAPDH is present in the scutellum during germination (Figure 3). At this stage the scutellar cells require energy, derived initially via glycolysis, for the synthesis of hydrolytic enzymes that are involved in the breakdown of grain storage components. In addition, the rich store of lipid in the cells of the scutellar epithelium (3) is mobilised via the gluconeogenic pathway to exportable energy forms that supply the early energy requirements of embryo growth. The cytosolic GAPDH is a key enzyme in both the glycolytic and gluconeogenic pathways, and the abundance of its $m R N A$ in the scutellar cells may reflect this fact.

\section{ACKNOWLEDGEMENTS}

The author is grateful to Prof. R. CERFF for providing the data concerning the mustard GAPDH clone and to ANDERS BRANDT and GEOFF FINCHER for providing the cDNA library. Special thanks are due to ANDERS BRANDT and Prof. Diter von WetTstein for critical reading of the manuscript. The work was supported by the Biomolecular Engineering Programme of the Commission of European Communities with Training Contract No. GBI0172-DK and Research Contract No. GBI-4024-DK to Prof. D. von WeTtSTeIN.

\section{REFERENCES}

1. Biesecker, G., J. I. Harris, J. C. Thierry, J. E. WALKER \& A. J. WONACOTT: Sequence and structure of D-glyceraldehyde-3-phosphate dehydrogenase from Bacillus stearothermophilus. Nature 266, 328-333 (1977)

2. Cerff, R. \& K. Kloppstech: Structural diversity and differential light control of $\mathrm{mRNAs}$ coding for angiosperm glyceraldehyde3-phosphate dehydrogenases. Proc. Nat. Acad. Sci. USA 79, 7624-7628 (1982)

3. GRAM, N. H.; The ultrastructure of germinating barley seeds. I. Changes in the scutellum and the aleurone layer in Nordal barley. Carlsberg Res. Commun. 47, 143-162 (1982)

4. Harris, J. I. \& R. N. Perham: Glyceraldehyde-3phosphate dehydrogenase from pig muscle. Nature 219, 1025-1028 (1968)

5. HARRIS, J. I. \& M. WATERS: Glyceraldehyde-3phosphate dehydrogenase. In The Enzymes XIII, 3rd edn. (ed. P. D. Boyer) 1-50 Academic Press, New York (1976)

6. Holland, J. P. \& M. J. Holland: The primary structure of a glyceraldehyde-3-phosphate dehy- 


\section{Second letter}

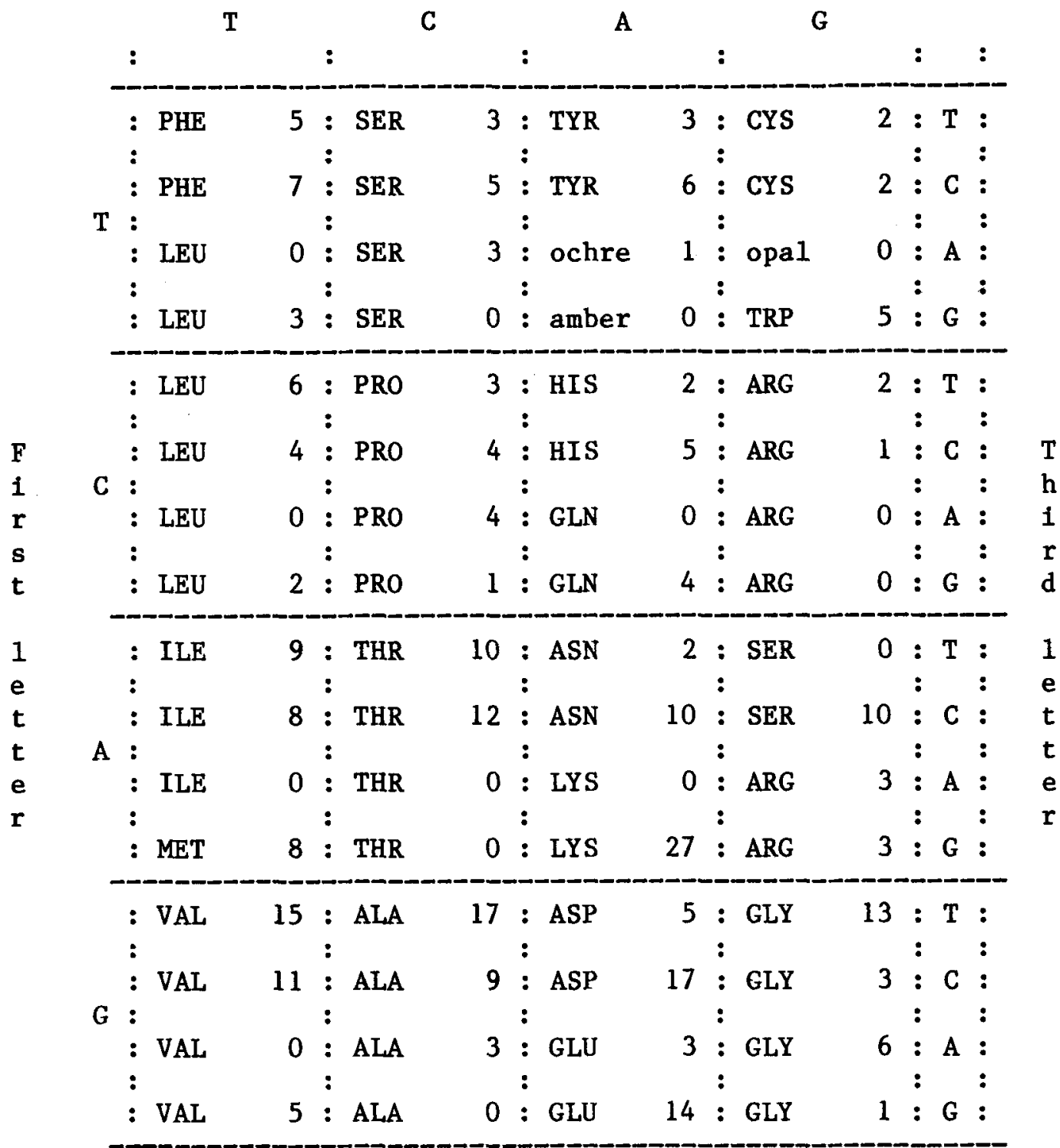

Figure 6. Codon usage of the gene for barley cytosolic GAPDH, as deduced from the cDNA sequence.

drogenase gene from Saccharomyces cerevisiae. J. Biol. Chem. 254, 9839-9845 (1979)

7. Hopp. H. E., S. K. RASmuSSEN \& A. BRANDT: Organisation and transcription of $B 1$ hordein genes in high lysine mutants of barley. Carlsberg Res. Commun, 48, 201-216 (1983)

8. Hu, N. \& J. MEssing: The making of strand-specific M13 probes. Gene 17, 271-277 (1982)
9. LoDISH, H. F.: Translational control of protein synthesis. Ann. Rev. Biochem. 45, 39-72 (1976)

10. Martin, W. \& R. CerfF: Prokaryotic origin of a plant nuclear gene: cDNA sequences for chloroplast and cytosolic glyceraldehyde-3-phosphate dehydrogenases from Sinapis alba. (1986)

11. Olsen, K. W., D. Moras, M. G. Rossman \& J. I. HARRIS: Sequence variability and structure of 
D-glyceraldehyde-3-phosphate dehydrogenase. J. Biol. Chem. 250, 9313-9321 (1975)

12. PiechaczyK, M..J. M. Blanchard, L. Marty, C. Dani, F. Panabieres, S. Sabouty, P. Fort \& P. JEANTEUR: Post-transcriptional regulation of glyceraldehyde-3-phosphate gene expression in rat tissues. Nucleic Acids Res. 12, 6951-6963 (1984)

13. Proudfoot, N. J.\& G.G. BrownleE: 3' Non-coding region sequences in eukaryotic $m R N A$. Nature 263, 211-214 (1976)

14. Rigby, P. W., M. Dieckmann, C. Rhodes \& P. BERG: Labelling deoxyribonucleic acid to high specific activity in vitro by nick translation with DNA polymerase I. J. Mol. Biol. 113, 237-251 (1977)

Accepted by H. KLENOW
15. Sanger. F., S. Nicklen \& A. R. Coulson: DNA sequencing with chain-termination inhibitors. Proc. Nat. Acad. Sci. USA 74, 5463-5467 (1977)

16. SOUTHERN, E. M.: Detection of specific sequences among DNA fragments separated by gel electrophoresis. J. Mol. Biol. 98,503-517 (1975)

17. Walker. J. E., A. J. WonacotT \& J. I. Harris: Heat stability of a tetrameric enzyme, D-glyceraldehyde-3-phosphate dehydrogenase. Eur. J. Biochem. 108, 581-586 (1980)

18. Wickens, M. P., G. N. Buell \& R. T. SChimke: Synthesis of double-stranded DNA complementary to lysozyme, ovomucoid and ovalbumin mRNAs. J. Biol. Chem. 253, 2483-2495 (1978) 